\title{
A LYAPUNOV FUNCTIONAL FOR A SYSTEM WITH A TIME-VARYING DELAY
}

\author{
JÓZEF DUDA \\ Department of Automatics \\ AGH University of Science and Technology, al. Mickiewicza 30, 30-059 Cracow, Poland \\ e-mail: jduda@agh.edu.pl
}

\begin{abstract}
The paper presents a method to determine a Lyapunov functional for a linear time-invariant system with an interval timevarying delay. The functional is constructed for the system with a time-varying delay with a given time derivative, which is calculated on the system trajectory. The presented method gives analytical formulas for the coefficients of the Lyapunov functional.
\end{abstract}

Keywords: Lyapunov functional, time delay system, LTI system.

\section{Introduction}

Lyapunov quadratic functionals are used to test the stability of systems, in computation of critical delay values for time delay systems, in computation of exponential estimates for solutions of time delay systems, in calculation of robustness bounds for uncertain time delay systems, in calculation of a quadratic performance index for the process of parametric optimization for time delay systems. We construct Lyapunov functionals for a system with a time delay with a given time derivative.

For the first time such a Lyapunov functional was introduced by Repin (1965) for the case of linear systems with one retarded-type delay. The author also delivered the procedure for determination of coefficients of the functional. Duda (1986) used the Lyapunov functional proposed by Repin for calculation of the value of a quadratic performance index in the process of parametric optimization for systems with a time delay, and extended the results to the case of a neutral type time delay system (Duda, 1988). He also presented a method of determining the Lyapunov functional for a linear dynamic system with two lumped retarded type time delays in the general case with non-commensurate delays and demonstrated a special case with commensurate delays in which the Lyapunov functional can be determined by solving a set of ordinary differential equations (Duda, 2010a), and showed a method of determining of the Lyapunov functional for linear dynamic system with two delays, both of retarded and neutral types (Duda, 2010b).

Infante and Castelan (1978) based the construc- tion of the Lyapunov functional on solution of a matrix differential-difference equation on a finite time interval. This solution satisfies symmetry and boundary conditions. Kharitonov and Zhabko (2003) extended the results of Infante and Castelan (1978) and proposed a procedure of construction of quadratic functionals for linear retarded type delay systems which could be used for robust stability analysis of time delay systems. This functional was expressed by means of a Lyapunov matrix, which depended on the fundamental matrix of a time delay system. Kharitonov (2005) extended some basic results obtained for the case of retarded type time delay systems to the case of neutral type time delay systems, and to neutral type time delay systems with a discrete and distributed delay (Kharitonov, 2008). Kharitonov and Hinrichsen (2004) used the Lyapunov matrix to derive exponential estimates for solutions of exponentially stable time delay systems. Kharitonov and Plischke (2006) formulated necessary and sufficient conditions for the existence and uniqueness of the delay Lyapunov matrix for the case of a retarded system with one delay.

A numerical scheme for construction of Lyapunov functionals was proposed by $\mathrm{Gu}$ (1997). This method starts with discretisation of the Lyapunov functional. The scheme is based on Linear Matrix Inequality (LMI) techniques. Fridman (2001) introduced Lyapunov-Krasovskii functionals for stability of linear retarded and neutral type systems with discrete and distributed delays, which were based on an equivalent descriptor form of the original system and obtained delay-dependent and delay-independent conditions in terms of LMIs. Ivanescu et al. (2003) pro- 
ceeded with delay-depended stability analysis for linear neutral systems, constructed the Lyapunov functional and derived sufficient delay-dependent conditions in terms of linear matrix inequalities.

Han (2004a) obtained a delay-dependent stability criterion for neutral systems with a time-varying discrete delay. This criterion was expressed in the form of an LMI and was obtained using the Lyapunov direct method. Han (2004b) investigated robust stability of uncertain neutral systems with discrete and distributed delays, which was based on descriptor model transformation and the decomposition technique, and formulated stability criteria in the form of LMIs. Han (2005a) considered the stability for linear neutral systems with norm-bounded uncertainties in all system matrices and derived a new delay-dependent stability criterion. Neither model transformation, nor the bounding technique for cross terms is involved in derivation of the stability criterion. Han (2005b) developed the discretized Lyapunov functional approach to investigation of the stability of linear neutral systems with mixed neutral and discrete delays. Stability criteria, which are applicable to linear neutral systems with both small and nonsmall discrete delays, are formulated in the form of LMIs.

Han (2009a) studied the problem of stability of linear time delay systems, both retarded and neutral types, using the discrete delay $N$-decomposition approach to derive some more general new discrete delay dependent stability criteria. Han (2009b) employed the delay-decomposition approach to derive some improved stability criteria for linear neutral systems and to deduce some sufficient conditions for the existence of the Lyapunov functional for a system with $k$-non-commensurate neutral time delays of a delayed state feedback controller, which ensure asymptotic stability and a prescribed $H_{1}$ performance level of the corresponding closed-loop system. Gu and Liu (2009) investigated the stability of coupled differentialfunctional equations using the discretized Lyapunov functional method and set forth the stability condition in the form of LMIs, suitable for numerical computation.

This paper presents a method of determining the Lyapunov functional for a linear time-invariant system with an interval time-varying delay. The functional is constructed for a system with a time-varying delay with a given time derivative which is calculated on the system trajectory. We assume that a time derivative of the Lyapunov functional is a quadratic form. This assumption enables calculation of the value of the integral quadratic performance index of parametric optimization of a system with a time delay. The presented method gives analytical formulas for the coefficients of the Lyapunov functional. The novelty of the result lies in the extension of the Repin method to the system with an interval time-varying delay. To the best of the author's knowledge, such extension has not been reported in the literature. An example illustrating that method is also presented.

\section{Problem formulation}

Let us consider a linear system with a time-varying delay, whose dynamics are described with the equation

$$
\begin{aligned}
\frac{\mathrm{d} x(t)}{\mathrm{d} t} & =A x(t)+B x_{t}(-\tau(t)), \\
x\left(t_{0}\right) & =x_{0} \in \mathbb{R}^{n}, \\
x_{t_{0}} & =\Phi \in W^{1,2}\left([-r, 0), \mathbb{R}^{n}\right),
\end{aligned}
$$

where we have $A, B \in \mathbb{R}^{n \times n} ; x(t) \in \mathbb{R}^{n} ; x_{t} \in$ $W^{1,2}\left([-r, 0), \mathbb{R}^{n}\right), x_{t}(\theta)=x(t+\theta) ; t \geq t_{0} ; \theta \in[-r, 0) ;$ $\tau(t)$ is a time-varying delay satisfying the condition $0 \leq$ $\tau(t) \leq r ; \mathrm{d} \tau(t) / \mathrm{d} t \neq 1 ; t \geq t_{0}$, where $r$ is a positive constant. $W^{1,2}\left([-r, 0), \mathbb{R}^{n}\right)$ is the space of all absolutely continuous functions $[-r, 0) \rightarrow \mathbb{R}^{n}$ with derivatives in $L^{2}\left([-r, 0), \mathbb{R}^{n}\right)$, the space of Lebesgue square integrable functions on the interval $[-r, 0)$ with values in $\mathbb{R}^{n}$.

The state of the system (1) is the vector

$$
S(t)=\left[\begin{array}{c}
x(t) \\
x_{t}
\end{array}\right] \quad \text { for } t \geq t_{0} .
$$

The state space is defined by the formula

$$
X=\mathbb{R}^{n} \times W^{1,2}\left([-r, 0), \mathbb{R}^{n}\right) .
$$

We define a Lyapunov functional, positive definite and differentiable, whose derivative computed on the trajectory of the system (1) is negative definite,

$$
\begin{aligned}
V(x(t), & \left.x_{t}, t\right) \\
= & x^{T}(t) \alpha(t) x(t) \\
& +\int_{-\tau(t)}^{0} x^{T}(t) \beta(\theta+\tau(t)) x_{t}(\theta) \mathrm{d} \theta \\
& +\int_{-\tau(t)}^{0} \int_{\theta}^{0} x_{t}^{T}(\theta) \delta(\theta+\tau(t), \sigma+\tau(t)) \\
& \cdot x_{t}(\sigma) \mathrm{d} \sigma \mathrm{d} \theta,
\end{aligned}
$$

for $t \geq t_{0}$, where $\alpha \in C^{1}\left(\left[t_{0}, \infty\right), \mathbb{R}^{n \times n}\right), \alpha(t)$ is positive definite, $\beta \in C^{1}\left([0, \tau(t)], \mathbb{R}^{n \times n}\right), \delta \in C^{1}\left(\Omega, \mathbb{R}^{n \times n}\right)$, $\Omega=\{(\theta, \sigma): \theta \in[0, \tau(t)], \sigma \in[\theta, 0]\} ; 0 \leq \tau(t) \leq r$. $C^{1}$ is the space of continuously differentiable functions.

The Lyapunov functional for a time-delay system with interval time-varying delay defined by (4) is more general than the ones found in the existing literature. To the best of the author's knowledge, such a functional has not been reported in the literature yet. 


\section{Determination of the coefficients of the Lyapunov functional}

We compute the derivative of the functional (4) on the trajectory of the system (1),

$$
\begin{aligned}
\frac{\mathrm{d} V}{V\left(x(t), x_{t}, t\right)} & \mathrm{d} t \\
= & x^{T}(t)\left[A^{T} \alpha(t)+\alpha(t) A+\frac{\mathrm{d} \alpha(t)}{\mathrm{d} t}\right. \\
& +\beta(\tau(t))] x(t)+x_{t}^{T}(-\tau(t))\left[B^{T}\left(\alpha(t)+\alpha^{T}(t)\right)\right. \\
& \left.+\beta^{T}(0)\left(\frac{\mathrm{d} \tau(t)}{\mathrm{d} t}-1\right)\right] x(t) \\
& +\int_{-\tau(t)}^{0} x^{T}(t)\left[A^{T} \beta(\theta+\tau(t))+\frac{\mathrm{d} \beta(\theta+\tau(t))}{\mathrm{d} t}\right. \\
& \left.-\frac{\mathrm{d} \beta(\theta+\tau(t))}{\mathrm{d} \theta}+\delta^{T}(\theta+\tau(t), \tau(t))\right] x_{t}(\theta) \mathrm{d} \theta \\
& +\int_{-\tau(t)}^{0} x_{t}^{T}(-\tau(t))\left[B^{T} \beta(\theta+\tau(t))\right. \\
& \left.+\delta(0, \theta+\tau(t))\left(\frac{\mathrm{d} \tau(t)}{\mathrm{d} t}-1\right)\right] x_{t}(\theta) \mathrm{d} \theta \\
& +\int_{-\tau(t)}^{0} \int_{\theta}^{0} x_{t}^{T}(\theta)\left[\frac{\mathrm{d} \delta(\theta+\tau(t), \sigma+\tau(t))}{\mathrm{d} t}\right. \\
& -\frac{\partial \delta(\theta+\tau(t), \sigma+\tau(t))}{\partial \theta} \\
& \left.-\frac{\partial \delta(\theta+\tau(t), \sigma+\tau(t))}{\partial \sigma}\right] x_{t}(\sigma) \mathrm{d} \sigma \mathrm{d} \theta
\end{aligned}
$$

for $t \geq t_{0}$, where

$$
\begin{gathered}
\alpha \in C^{1}\left(\left[t_{0}, \infty\right), \mathbb{R}^{n \times n}\right) ; \\
\beta \in C^{1}\left([0, \tau(t)], \mathbb{R}^{n \times n}\right), \quad \delta \in C^{1}\left(\Omega, \mathbb{R}^{n \times n}\right), \\
\Omega=\{(\theta, \sigma): \theta \in[0, \tau(t)], \sigma \in[\theta, 0]\}, \\
0 \leq \tau(t) \leq r .
\end{gathered}
$$

We identify the coefficients of the Lyapunov functional (4) assuming that the derivative (5) satisfies the relationship

$$
\frac{\mathrm{d} V\left(x(t), x_{t}, t\right)}{\mathrm{d} t}=-x^{T}(t) W x(t) \quad \text { for } t \geq t_{0},
$$

where $W \in \mathbb{R}^{n \times n}$ is a positive definite matrix.

When the relationship (6) holds and we know the
Lyapunov functional, we can easily determine the value of the square performance index since

$$
J=\int_{t_{0}}^{\infty} x^{T}(t) W x(t) \mathrm{d} t=V\left(x_{0}, \Phi, t_{0}\right) .
$$

From Eqns. (5) and (6) we obtain the set of equations

$$
\begin{gathered}
A^{T} \alpha(t)+\alpha(t) A+\frac{\mathrm{d} \alpha(t)}{\mathrm{d} t}+\beta(\tau(t))=-W, \\
B^{T}\left(\alpha(t)+\alpha^{T}(t)\right)+\beta^{T}(0)\left(\frac{\mathrm{d} \tau(t)}{\mathrm{d} t}-1\right)=0, \\
A^{T} \beta(\theta+\tau(t))+\frac{\mathrm{d} \beta(\theta+\tau(t))}{\mathrm{d} t} \\
-\frac{\mathrm{d} \beta(\theta+\tau(t))}{\mathrm{d} \theta}+\delta^{T}(\theta+\tau(t), \tau(t))=0,
\end{gathered}
$$

$$
\begin{aligned}
& B^{T} \beta(\theta+\tau(t)) \\
& \quad+\delta(0, \theta+\tau(t))\left(\frac{\mathrm{d} \tau(t)}{\mathrm{d} t}-1\right)=0,
\end{aligned}
$$

$$
\begin{gathered}
\frac{\mathrm{d} \delta(\theta+\tau(t), \sigma+\tau(t))}{\mathrm{d} t}-\frac{\partial \delta(\theta+\tau(t), \sigma+\tau(t))}{\partial \theta} \\
-\frac{\partial \delta(\theta+\tau(t), \sigma+\tau(t))}{\partial \sigma}=0,
\end{gathered}
$$

for $t \geq t_{0}, \theta \in[-\tau(t), 0], \sigma \in[\theta, 0]$, where $0 \leq \tau(t) \leq r$.

We introduce new variables,

$$
\begin{aligned}
& \xi=\theta+\tau(t), \\
& \eta=\sigma+\tau(t),
\end{aligned}
$$

and calculate the derivatives,

$$
\begin{gathered}
\frac{\mathrm{d} \delta(\theta+\tau(t), \sigma+\tau(t))}{\mathrm{d} t}=\frac{\mathrm{d} \delta(\xi, \eta)}{\mathrm{d} t} \\
=\frac{\partial \delta(\xi, \eta)}{\partial \xi} \frac{\mathrm{d} \tau(t)}{\mathrm{d} t}+\frac{\partial \delta(\xi, \eta)}{\partial \eta} \frac{\mathrm{d} \tau(t)}{\mathrm{d} t}, \\
\frac{\partial \delta(\theta+\tau(t), \sigma+\tau(t))}{\partial \theta}=\frac{\partial \delta(\xi, \eta)}{\partial \theta}=\frac{\partial \delta(\xi, \eta)}{\partial \xi}, \\
\frac{\partial \delta(\theta+\tau(t), \sigma+\tau(t))}{\partial \sigma}=\frac{\partial \delta(\xi, \eta)}{\partial \sigma}=\frac{\partial \delta(\xi, \eta)}{\partial \eta}, \\
\frac{\mathrm{d} \beta(\theta+\tau(t))}{\mathrm{d} t}=\frac{d \beta(\xi)}{d \xi} \frac{\partial \xi}{\partial t}=\frac{\mathrm{d} \beta(\xi)}{\mathrm{d} \xi} \frac{\mathrm{d} \tau(t)}{\mathrm{d} t}, \\
\frac{\mathrm{d} \beta(\theta+\tau(t))}{\mathrm{d} \theta}=\frac{\mathrm{d} \beta(\xi)}{\mathrm{d} \xi} \frac{\partial \xi}{\partial \theta}=\frac{\mathrm{d} \beta(\xi)}{\mathrm{d} \xi} .
\end{gathered}
$$


The formula (12) takes the form

$$
\frac{\partial \delta(\xi, \eta)}{\partial \xi}+\frac{\partial \delta(\xi, \eta)}{\partial \eta}=0
$$

for $t \geq t_{0}, \theta \in[-\tau(t), 0], \sigma \in[\theta, 0], \xi \in[0, \tau(t)], \eta \in$ $[\xi, \tau(t)]$, where $0 \leq \tau(t) \leq r$.

The solution of Eqn. (12) is given by

$$
\begin{aligned}
\delta(\theta+\tau(t), \sigma+\tau(t)) & =\delta(\xi, \eta) \\
& =f(\xi-\eta)=f(\theta-\sigma)
\end{aligned}
$$

for $t \geq t_{0}, \theta \in[-\tau(t), 0], \sigma \in[\theta, 0], 0 \leq \tau(t) \leq r$, where $f \in C^{1}\left([-r, r], \mathbb{R}^{n \times n}\right)$.

Taking 21 into account, from Eqn. (11) we get the relationship

$$
\begin{aligned}
\delta(0, \theta+\tau(t)) & =f(-\tau(t)-\theta) \\
& =\left(1-\frac{\mathrm{d} \tau(t)}{\mathrm{d} t}\right)^{-1} B^{T} \beta(\theta+\tau(t)) .
\end{aligned}
$$

Hence

$$
f(\xi)=\left(1-\frac{\mathrm{d} \tau(t)}{\mathrm{d} t}\right)^{-1} B^{T} \beta(-\xi)
$$

for $\xi \in[0, \tau(t)]$, where $0 \leq \tau(t) \leq r$.

From 21) we obtain

$$
\begin{aligned}
\delta^{T}(\theta+\tau(t), \tau(t)) & =f^{T}(\theta) \\
& =\left(1-\frac{\mathrm{d} \tau(t)}{\mathrm{d} t}\right)^{-1} \beta^{T}(-\theta) B .
\end{aligned}
$$

We substitute the term (24) into (10) and obtain

$$
\begin{aligned}
& A^{T} \beta(\theta+\tau(t))+\frac{\mathrm{d} \beta(\theta+\tau(t))}{\mathrm{d} t} \\
& -\frac{\mathrm{d} \beta(\theta+\tau(t))}{\mathrm{d} \theta}+\left(1-\frac{d \tau(t)}{\mathrm{d} t}\right)^{-1} \beta^{T}(-\theta) B=0 .
\end{aligned}
$$

Taking (13), 18) and 196 into account from Eqn. 25) we get the relationship

$$
\begin{aligned}
\frac{\mathrm{d} \beta(\xi)}{\mathrm{d} \xi}= & -\left(\frac{\mathrm{d} \tau(t)}{\mathrm{d} t}-1\right)^{-1} A^{T} \beta(\xi) \\
& +\left(\frac{\mathrm{d} \tau(t)}{\mathrm{d} t}-1\right)^{-2} \beta^{T}(-\xi+\tau(t)) B
\end{aligned}
$$

for $\xi \in[0, \tau(t)]$, where $0 \leq \tau(t) \leq r$.

We calculate the derivative of the term $\beta(-\xi+\tau(t))$ with respect to $\xi$ using the relationship (26). We get

$$
\begin{aligned}
\frac{\mathrm{d} \beta(-\xi+\tau(t))}{\mathrm{d} \xi} \\
=-\left(\frac{\mathrm{d} \tau(t)}{\mathrm{d} t}-1\right)^{-2} \beta^{T}(\xi) B \\
\quad+\left(\frac{\mathrm{d} \tau(t)}{\mathrm{d} t}-1\right)^{-1} A^{T} \beta(-\xi+\tau(t))
\end{aligned}
$$

for $\xi \in[0, \tau(t)]$, where $0 \leq \tau(t) \leq r$.

In such a way, we get the set of differential equations,

$$
\left\{\begin{aligned}
\frac{\mathrm{d} \beta(\xi)}{\mathrm{d} \xi}=- & \left(\frac{\mathrm{d} \tau(t)}{\mathrm{d} t}-1\right)^{-1} A^{T} \beta(\xi) \\
& +\left(\frac{\mathrm{d} \tau(t)}{\mathrm{d} t}-1\right)^{-2} \beta^{T}(-\xi+\tau(t)) B \\
\frac{\mathrm{d} \beta(-\xi+\tau(t))}{\mathrm{d} \xi} & =-\left(\frac{\mathrm{d} \tau(t)}{\mathrm{d} t}-1\right)^{-2} \beta^{T}(\xi) B \\
& +\left(\frac{\mathrm{d} \tau(t)}{\mathrm{d} t}-1\right)^{-1} A^{T} \beta(-\xi+\tau(t))
\end{aligned}\right.
$$

for each fixed $t \geq t_{0}, \xi \in[0, \tau(t)]$, where $0 \leq \tau(t) \leq r$. with the initial conditions $\beta(0)$ and $\beta(\tau(t))$.

There is a relationship between $\beta(\xi)$ and $\beta(-\xi+$ $\tau(t))$ such that

$$
\left.\beta(\xi)\right|_{\xi=\frac{\tau(t)}{2}}=\left.\beta(-\xi+\tau(t))\right|_{\xi=\frac{\tau(t)}{2}} .
$$

We calculate the derivative of Eqn. (9) with respect to $t$,

$$
\begin{aligned}
B^{T}\left(\frac{\mathrm{d} \alpha(t)}{\mathrm{d} t}+\frac{\mathrm{d} \alpha^{T}(t)}{\mathrm{d} t}\right) & +\frac{\mathrm{d} \beta^{T}(0)}{\mathrm{d} t}\left(\frac{\mathrm{d} \tau(t)}{\mathrm{d} t}-1\right) \\
& +\beta^{T}(0) \frac{\mathrm{d}^{2} \tau(t)}{\mathrm{d} t^{2}}=0 .
\end{aligned}
$$

From Eqn. (26) it follows that

$$
\begin{aligned}
\frac{\mathrm{d} \beta^{T}(0)}{\mathrm{d} t}= & -\frac{\mathrm{d} \tau(t)}{\mathrm{d} t}\left(\frac{\mathrm{d} \tau(t)}{\mathrm{d} t}-1\right)^{-1} \beta^{T}(0) A \\
& +\frac{\mathrm{d} \tau(t)}{\mathrm{d} t}\left(\frac{\mathrm{d} \tau(t)}{\mathrm{d} t}-1\right)^{-2} B^{T} \beta(\tau(t)) .
\end{aligned}
$$

From Eqn. (8) we obtain

$\frac{\mathrm{d} \alpha(t)}{\mathrm{d} t}=-A^{T} \alpha(t)-\alpha(t) A-\beta(\tau(t))-W$.

We substitute the terms (31) and (32) into Eqn. (30), 
and, after calculations, we get

$$
\begin{aligned}
& B^{T}\left[A^{T}\left(\alpha(t)+\alpha^{T}(t)\right)+\left(\alpha(t)+\alpha^{T}(t)\right) A\right] \\
& +\beta^{T}(0)\left(\frac{\mathrm{d} \tau(t)}{\mathrm{d} t} A-\frac{\mathrm{d}^{2} \tau(t)}{\mathrm{d} t^{2}} I\right) \\
& -\left(\frac{\mathrm{d} \tau(t)}{\mathrm{d} t}-1\right)^{-1} B^{T} \beta(\tau(t))+B^{T} \beta^{T}(\tau(t)) \\
& =-B^{T}\left(W+W^{T}\right) .
\end{aligned}
$$

The matrix $\alpha(t)$ and the initial conditions of the system (28) are obtained by solving Eqns. (33), (9) and (29):

$$
\begin{aligned}
& B^{T}\left[A^{T}\left(\alpha(t)+\alpha^{T}(t)\right)+\left(\alpha(t)+\alpha^{T}(t)\right) A\right] \\
& +\beta^{T}(0)\left(\frac{\mathrm{d} \tau(t)}{\mathrm{d} t} A-\frac{\mathrm{d}^{2} \tau(t)}{\mathrm{d} t^{2}} I\right) \\
& -\left(\frac{\mathrm{d} \tau(t)}{\mathrm{d} t}-1\right)^{-1} B^{T} \beta(\tau(t))+B^{T} \beta^{T}(\tau(t)) \\
& =-B^{T}\left(W+W^{T}\right), \\
& B^{T}\left(\alpha(t)+\alpha^{T}(t)\right)+\beta^{T}(0)\left(\frac{\mathrm{d} \tau(t)}{\mathrm{d} t}-1\right)=0, \\
& \left.\beta(\xi)\right|_{\xi=\frac{\tau(t)}{2}}=\left.\beta(-\xi+\tau(t))\right|_{\xi=\frac{\tau(t)}{2}} .
\end{aligned}
$$

Having the solution of the set of differential equations (28) and taking (13), (21) and (23) into account, we obtain the matrices

$$
\begin{gathered}
\beta(\theta+\tau(t))=\left.\beta(\xi)\right|_{\xi=\theta+\tau(t)}, \\
\delta(\theta+\tau(t), \sigma+\tau(t)) \\
=\left(1-\frac{\mathrm{d} \tau(t)}{\mathrm{d} t}\right)^{-1} B^{T} \beta(\sigma-\theta)
\end{gathered}
$$

for $t \geq t_{0}, \theta \in[-\tau(t), 0], \sigma \in[\theta, 0]$ where $0 \leq \tau(t) \leq r$.

In this way, we have obtained all the parameters of the Lyapunov functional (4).

\section{Example}

Let us consider the system described by

$$
\begin{aligned}
\frac{\mathrm{d} x(t)}{\mathrm{d} t} & =a x(t)+b x_{t}(-\tau(t)), \\
x\left(t_{0}\right) & =x_{0} \in \mathbb{R}, \\
x_{t_{0}} & =\Phi,
\end{aligned}
$$

$t \geq t_{0} ; x_{t}, \Phi \in W^{1,2}([-r, 0), \mathbb{R}) ; x(t) \in \mathbb{R} ; a, b \in \mathbb{R} ;$ $\tau(t)$ is a time-varying delay satisfying the condition $0 \leq$ $\tau(t) \leq r ; \mathrm{d} \tau(t) / \mathrm{d} t \neq 1 ; t \geq t_{0}$, where $r$ is positive constant.

The Lyapunov functional is given by

$$
\begin{aligned}
& V\left(x(t), x_{t}, t\right) \\
& =\alpha(t) x^{2}(t)+\int_{-\tau(t)}^{0} \beta(\theta+\tau(t)) x(t) x_{t}(\theta) \mathrm{d} \theta \\
& +\int_{-\tau(t)}^{0} \int_{\theta}^{0} \delta(\theta+\tau(t), \sigma+\tau(t)) x_{t}(\theta) x_{t}(\sigma) \mathrm{d} \sigma \mathrm{d} \theta .
\end{aligned}
$$

We obtain the coefficients of the functional as below.

Equation (28) takes the form

$$
\begin{aligned}
& {\left[\begin{array}{c}
\frac{\mathrm{d} \beta(\xi)}{\mathrm{d} \theta} \\
\frac{\mathrm{d} \beta(-\xi+\tau(t))}{\mathrm{d} \theta}
\end{array}\right]} \\
& =\left[\begin{array}{cc}
\frac{a}{1-\frac{\mathrm{d} \tau(t)}{\mathrm{d} t}} & \frac{b}{\left(1-\frac{\mathrm{d} \tau(t)}{\mathrm{d} t}\right)^{2}} \\
-\frac{b}{\left(1-\frac{\mathrm{d} \tau(t)}{\mathrm{d} t}\right)^{2}} & -\frac{a}{1-\frac{\mathrm{d} \tau(t)}{\mathrm{d} t}}
\end{array}\right] \\
& \times\left[\begin{array}{c}
\beta(\xi) \\
\beta(-\xi+\tau(t))
\end{array}\right]
\end{aligned}
$$

for $t \geq t_{0}, \xi \in[0, \tau(t)]$, where $0 \leq \tau(t) \leq r$.

The fundamental matrix of the differential equation (41) is given by

$$
=\left[\begin{array}{c}
\operatorname{ch} \lambda \xi+\frac{a}{\lambda\left(1-\frac{\mathrm{d} \tau(t)}{\mathrm{d} t}\right)} \operatorname{sh} \lambda \xi \\
-\frac{b}{\lambda\left(1-\frac{\mathrm{d} \tau(t)}{\mathrm{d} t}\right)^{2}} \operatorname{sh} \lambda \xi
\end{array}\right.
$$

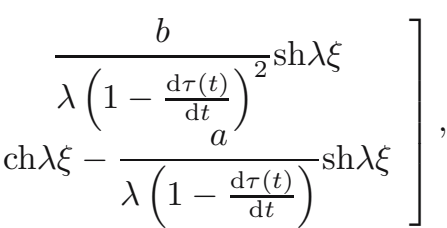


where

$$
\lambda=\frac{\sqrt{a^{2}\left(1-\frac{\mathrm{d} \tau(t)}{\mathrm{d} t}\right)^{2}-b^{2}}}{\left(1-\frac{\mathrm{d} \tau(t)}{\mathrm{d} t}\right)^{2}}
$$

Hence

$$
\left[\begin{array}{c}
\beta(\xi) \\
\beta(-\xi+\tau(t))
\end{array}\right]=Q(\xi)\left[\begin{array}{c}
\beta(0) \\
\beta(\tau(t))
\end{array}\right]
$$

for $t \geq t_{0}, \xi \in[0, \tau(t)]$, where $0 \leq \tau(t) \leq r$.

We need the initial conditions of the set of differential equations 41 to obtain

$$
\begin{gathered}
\beta(\theta+\tau(t))=\left.\beta(\xi)\right|_{\xi=\theta+\tau(t)}, \\
\delta(\theta+\tau(t), \sigma+\tau(t))=\left(1-\frac{\mathrm{d} \tau(t)}{\mathrm{d} t}\right)^{-1} b \beta(\sigma-\theta) .
\end{gathered}
$$

for

$$
t \geq t_{0}, \quad \theta \in[-\tau(t), 0], \quad \sigma \in[\theta, 0],
$$

where

$$
0 \leq \tau(t) \leq r
$$

The initial conditions of the differential equation (41) and the coefficient $\alpha(t)$ are obtained by solving the set of equations (34)-(36), which take the following form:

$$
\begin{gathered}
4 a b \alpha(t)+\left(a \frac{\mathrm{d} \tau(t)}{\mathrm{d} t}-\frac{\mathrm{d}^{2} \tau(t)}{\mathrm{d} t^{2}}\right) \beta(0) \\
+\left(1-\frac{1}{\frac{\mathrm{d} \tau(t)}{\mathrm{d} t}-1}\right) b \beta(\tau(t))=-2 b w \\
2 b \alpha(t)+\left(\frac{\mathrm{d} \tau(t)}{\mathrm{d} t}-1\right) \beta(0)=0, \\
p_{1} \beta(0)+p_{2} \beta(\tau(t))=0,
\end{gathered}
$$

where

$$
\begin{aligned}
p_{1}= & \operatorname{ch} \frac{\lambda \tau(t)}{2}+\left(\frac{a}{\lambda\left(1-\frac{\mathrm{d} \tau(t)}{\mathrm{d} t}\right)}\right. \\
& \left.+\frac{b}{\lambda\left(1-\frac{\mathrm{d} \tau(t)}{\mathrm{d} t}\right)^{2}}\right) \operatorname{sh} \frac{\lambda \tau(t)}{2}, \\
p_{2}= & -\operatorname{ch} \frac{\lambda \tau(t)}{2} \\
& +\left(\frac{a}{\lambda\left(1-\frac{\mathrm{d} \tau(t)}{\mathrm{d} t}\right)}+\frac{b}{\lambda\left(1-\frac{\mathrm{d} \tau(t)}{\mathrm{d} t}\right)^{2}}\right) \operatorname{sh} \frac{\lambda \tau(t)}{2} .
\end{aligned}
$$

Figures 1 and 2 show graphs of the functions $\alpha(t)$

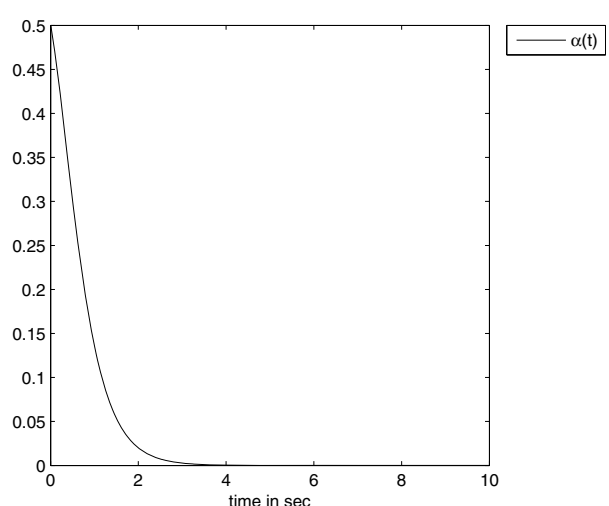

Fig. 1. Parameter $\alpha(t)$.

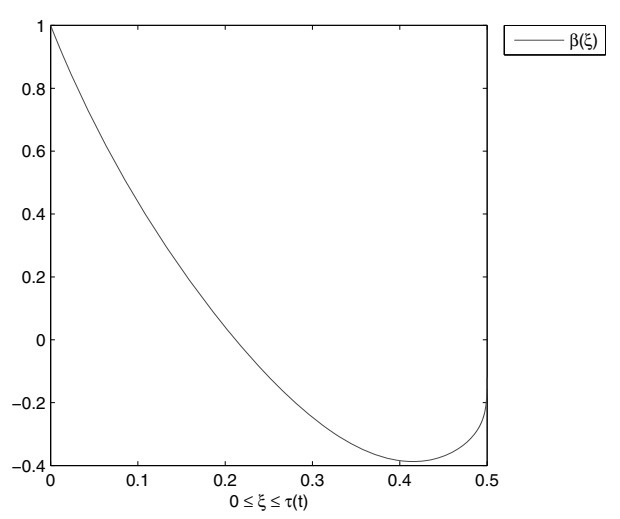

Fig. 2. Parameter $\beta(\xi)$.

and $\beta(\xi)$, obtained with a Matlab code, for the given values of parameters of the system (39).

$$
\begin{gathered}
a=-1, \quad b=-0.5, \quad w=1 \\
\tau(t)=r\left(1-\exp \left(-\frac{t}{T}\right)\right), \quad r=0.5, \quad T=1 .
\end{gathered}
$$

The parameter $\alpha(t)$ is positive for $r \leq 1.25$, so we have obtained a region of stability for the system (39).

Let us consider the system described by equation

$$
\left\{\begin{aligned}
{\left[\begin{array}{l}
\frac{\mathrm{d} x_{1}(t)}{\mathrm{d} t} \\
\frac{\mathrm{d} x_{2}(t)}{\mathrm{d} t}
\end{array}\right]=} & {\left[\begin{array}{ll}
a_{11} & a_{12} \\
a_{21} & a_{22}
\end{array}\right]\left[\begin{array}{l}
x_{1}(t) \\
x_{2}(t)
\end{array}\right] } \\
& +\left[\begin{array}{ll}
b_{11} & b_{12} \\
b_{21} & b_{22}
\end{array}\right]\left[\begin{array}{l}
x_{t_{1}}(-\tau(t)) \\
x_{t_{2}}(-\tau(t))
\end{array}\right], \\
{\left[\begin{array}{l}
x_{1}\left(t_{0}\right) \\
x_{2}\left(t_{0}\right)
\end{array}\right]=} & {\left[\begin{array}{l}
x_{10} \\
x_{20}
\end{array}\right] } \\
{\left[\begin{array}{l}
x_{t_{1}}(\theta) \\
x_{t_{2}}(\theta)
\end{array}\right]=} & {\left[\begin{array}{l}
\Phi_{1}(\theta) \\
\Phi_{2}(\theta)
\end{array}\right] . }
\end{aligned}\right.
$$


The Lyapunov functional is defined by

$$
\begin{aligned}
& V\left(x(t), x_{t}, t\right) \\
& =\left[\begin{array}{ll}
x_{1}(t) & \left.x_{2}(t)\right]\left[\begin{array}{ll}
\alpha_{11}(t) & \alpha_{12}(t) \\
\alpha_{21}(t) & \alpha_{22}(t)
\end{array}\right]\left[\begin{array}{l}
x_{1}(t) \\
x_{2}(t)
\end{array}\right] \\
& +\int_{-\tau(t)}^{0}\left[\begin{array}{ll}
x_{1}(t) & x_{2}(t)
\end{array}\right] \\
& \times\left[\begin{array}{ll}
\beta_{11}(\theta+\tau(t)) & \beta_{12}(\theta+\tau(t)) \\
\beta_{21}(\theta+\tau(t)) & \beta_{22}(\theta+\tau(t))
\end{array}\right]\left[\begin{array}{l}
x_{t_{1}}(\theta) \\
x_{t_{2}}(\theta)
\end{array}\right] \mathrm{d} \theta \\
+ & \left.\int_{-\tau(t)}^{0} \begin{array}{l}
0 \\
\int_{\theta}^{0}
\end{array}\right] \\
\times & {\left[\begin{array}{c}
\delta_{t_{1}}(\theta) \\
\delta_{t_{2}}(\theta)
\end{array}\right]} \\
\delta_{21}(\theta+\tau(t), \sigma+\tau(t)) & \delta_{12}(\theta+\tau(t), \sigma+\tau(t)) \\
\delta_{22}(\theta+\tau(t), \sigma+\tau(t))
\end{array}\right] \\
& \times\left[\begin{array}{l}
x_{t_{1}}(\sigma) \\
x_{t_{2}}(\sigma)
\end{array}\right] \mathrm{d} \sigma \mathrm{d} \theta
\end{aligned}
$$

for

$$
t \geq t_{0}, \quad \theta \in[-\tau(t), 0], \quad \sigma \in[\theta, 0],
$$

where

$$
0 \leq \tau(t) \leq r
$$

The set of equations (28) becomes

$$
\begin{aligned}
\frac{\mathrm{d}}{\mathrm{d} \xi}\left[\begin{array}{c}
\operatorname{col} \beta(\xi) \\
\operatorname{col} \beta(-\xi+\tau(t))
\end{array}\right] & =Q\left[\begin{array}{c}
\operatorname{col} \beta(\xi) \\
\operatorname{col} \beta(-\xi+\tau(t))
\end{array}\right]
\end{aligned}
$$

for

$$
\xi \in[0, \tau(t)]
$$

where

$$
\begin{gathered}
0 \leq \tau(t) \leq r, \\
Q=\left[\begin{array}{ll}
-Q_{1} & Q_{2}
\end{array}\right],
\end{gathered}
$$

and $Q_{1}$ and $Q_{2}$ are given by (56) and (57), respectively, while

$$
\left[\begin{array}{c}
\operatorname{col} \beta(\xi) \\
\operatorname{col} \beta(-\xi+\tau(t))
\end{array}\right]=e^{Q \xi}\left[\begin{array}{c}
\operatorname{col} \beta(0) \\
\operatorname{col} \beta(\tau(t))
\end{array}\right]
$$

for $\xi \in[0, \tau(t)]$, where $0 \leq \tau(t) \leq r$.
We introduce

$$
e^{Q \tau(t) / 2}=\left[\begin{array}{llllllll}
p_{11} & p_{12} & p_{13} & p_{14} & p_{15} & p_{16} & p_{17} & p_{18} \\
p_{21} & p_{22} & p_{23} & p_{24} & p_{25} & p_{26} & p_{27} & p_{28} \\
p_{31} & p_{32} & p_{33} & p_{34} & p_{35} & p_{36} & p_{37} & p_{38} \\
p_{41} & p_{42} & p_{43} & p_{44} & p_{45} & p_{46} & p_{47} & p_{48} \\
p_{51} & p_{52} & p_{53} & p_{54} & p_{55} & p_{56} & p_{57} & p_{58} \\
p_{61} & p_{62} & p_{63} & p_{64} & p_{65} & p_{66} & p_{67} & p_{68} \\
p_{71} & p_{72} & p_{73} & p_{74} & p_{75} & p_{76} & p_{77} & p_{78} \\
p_{81} & p_{82} & p_{83} & p_{84} & p_{85} & p_{86} & p_{87} & p_{88}
\end{array}\right] .
$$

Now we give the formulas for determination of the set of the initial conditions of the equation (54) and the matrix $\alpha$ :

$$
\begin{aligned}
& Z\left[\begin{array}{c}
\operatorname{col} \alpha(t) \\
\operatorname{col} \beta(0) \\
\operatorname{col} \beta(\tau(t))
\end{array}\right] \\
& \quad=\left[\begin{array}{c}
-2 b_{11} w_{11}-b_{21} w_{12}-b_{21} w_{21} \\
-b_{11} w_{12}-b_{11} w_{21}-2 b_{21} w_{22} \\
-2 b_{12} w_{11}-b_{22} w_{12}-b_{22} w_{21} \\
-b_{12} w_{12}-b_{12} w_{21}-2 b_{22} w_{22} \\
0_{(8,1)}
\end{array}\right],
\end{aligned}
$$

where

$$
\begin{aligned}
Z & =\left[\begin{array}{ccc}
D & Z_{12} & Z_{13} \\
Z_{21} & Z_{22} & 0_{(4,4)} \\
0_{(4,4)} & Z_{32} & Z_{33}
\end{array}\right], \\
D & =\left[\begin{array}{llll}
d_{11} & d_{12} & d_{13} & d_{14} \\
d_{21} & d_{22} & d_{23} & d_{24} \\
d_{31} & d_{31} & d_{33} & d_{34} \\
d_{41} & d_{42} & d_{43} & d_{44}
\end{array}\right],
\end{aligned}
$$

$$
\begin{aligned}
& d_{11}=4 a_{11} b_{11}+2 a_{12} b_{21}, \\
& d_{12}=d_{13}=2 a_{21} b_{11}+a_{22} b_{21}+a_{11} b_{21}, \\
& d_{14}=2 a_{21} b_{21}, \\
& d_{21}=2 a_{12} b_{11}, \\
& d_{22}=d_{23}=a_{11} b_{11}+a_{22} b_{11}+2 a_{12} b_{21}, \\
& d_{24}=2 a_{21} b_{11}+4 a_{22} b_{21}, \\
& d_{31}=4 a_{11} b_{12}+2 a_{12} b_{22}, \\
& d_{32}=d_{33}=2 a_{21} b_{12}+a_{22} b_{22}+a_{11} b_{22}, \\
& d_{34}=2 a_{21} b_{22}, \\
& d_{41}=2 a_{12} b_{12}, \\
& d_{42}=d_{43}=a_{11} b_{12}+a_{22} b_{12}+2 a_{12} b_{22}, \\
& d_{44}=2 a_{21} b_{12}+4 a_{22} b_{22}, \\
& Z_{12}=\left[Z_{12}^{1} Z_{12}^{2}\right],
\end{aligned}
$$




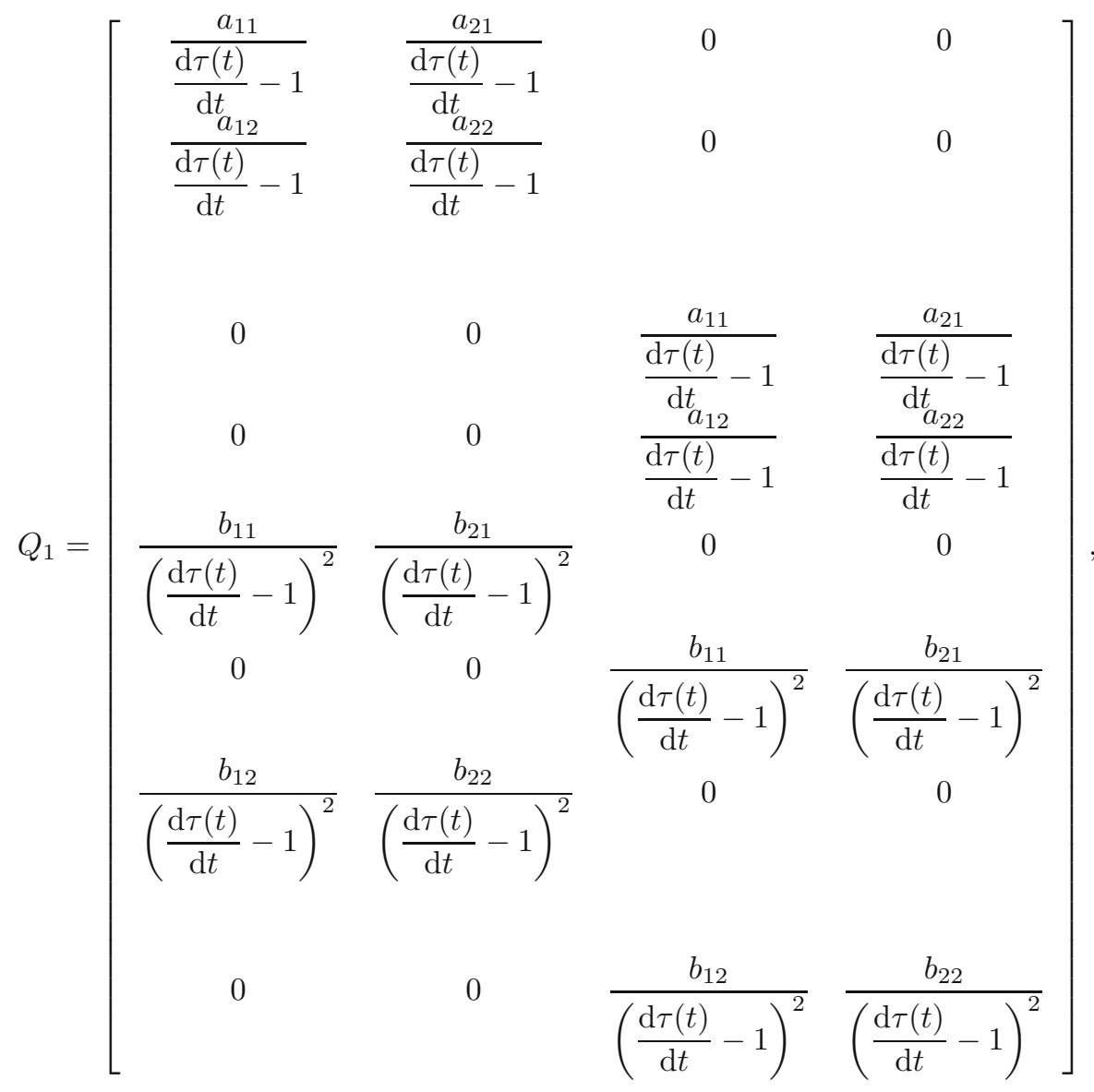

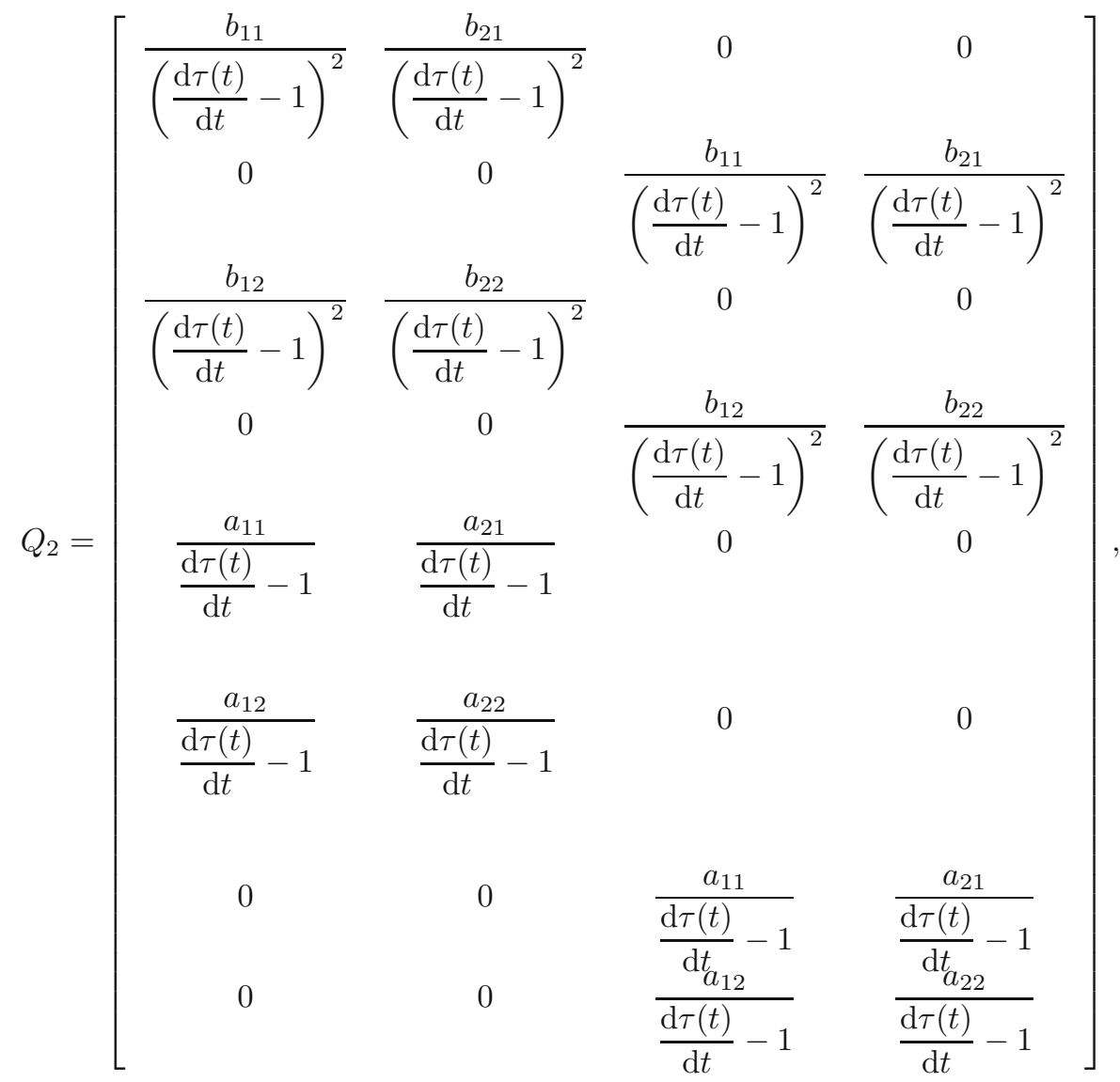




$$
Z_{12}^{1}=\left[\begin{array}{cc}
\frac{\mathrm{d} \tau(t)}{\mathrm{d} t} a_{11}-\frac{\mathrm{d}^{2} \tau(t)}{\mathrm{d} t^{2}} & \frac{\mathrm{d} \tau(t)}{\mathrm{d} t} a_{21} \\
\frac{\mathrm{d} \tau(t)}{\mathrm{d} t} a_{12} & \frac{\mathrm{d} \tau(t)}{\mathrm{d} t} a_{22}-\frac{\mathrm{d}^{2} \tau(t)}{\mathrm{d} t^{2}} \\
0 & 0 \\
0 & 0
\end{array}\right],
$$

$$
Z_{12}^{2}=\left[\begin{array}{cc}
0 & 0 \\
0 & 0 \\
\frac{\mathrm{d} \tau(t)}{\mathrm{d} t} a_{11}-\frac{\mathrm{d}^{2} \tau(t)}{\mathrm{d} t^{2}} & \frac{\mathrm{d} \tau(t)}{\mathrm{d} t} a_{21} \\
\frac{\mathrm{d} \tau(t)}{\mathrm{d} t} a_{12} & \frac{\mathrm{d} \tau(t)}{\mathrm{d} t} a_{22}-\frac{\mathrm{d}^{2} \tau(t)}{\mathrm{d} t^{2}}
\end{array}\right]_{(77)}
$$

$$
Z_{13}=\left[Z_{13}^{1} Z_{13}^{2}\right]
$$

$$
Z_{13}^{1}=\left[\begin{array}{cc}
b_{11}\left(1-\frac{1}{\frac{\mathrm{d} \tau(t)}{\mathrm{d} t}-1}\right) & -\frac{b_{21}}{\frac{\mathrm{d} \tau(t)}{\mathrm{d} t}-1} \\
0 & b_{11} \\
b_{12}\left(1-\frac{1}{\frac{\mathrm{d} \tau(t)}{\mathrm{d} t}-1}\right) & -\frac{b_{22}}{\frac{\mathrm{d} \tau(t)}{\mathrm{d} t}-1} \\
0 & b_{12}
\end{array}\right],
$$

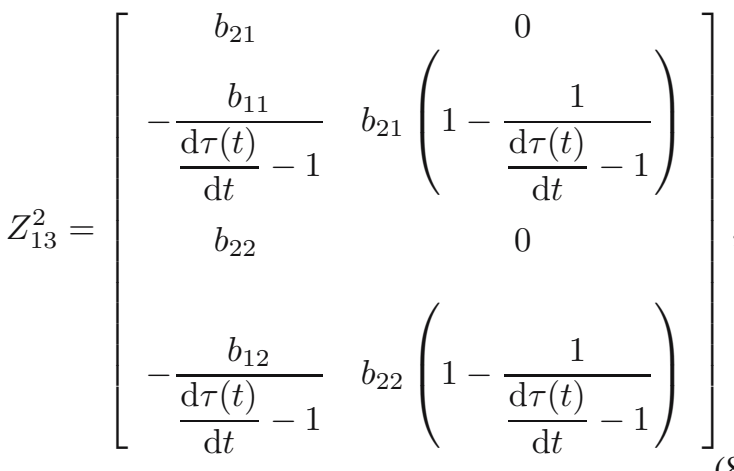

$$
\begin{aligned}
& Z_{21}=\left[\begin{array}{cccc}
2 b_{11} & b_{21} & b_{21} & 0 \\
0 & b_{11} & b_{11} & 2 b_{21} \\
2 b_{12} & b_{22} & b_{22} & 0 \\
0 & b_{12} & b_{12} & 2 b_{22}
\end{array}\right]
\end{aligned}
$$

$Z_{22}$

$$
=\left[\begin{array}{cccc}
\frac{\mathrm{d} \tau(t)}{\mathrm{d} t}-1 & 0 & 0 & 0 \\
0 & \frac{\mathrm{d} \tau(t)}{\mathrm{d} t}-1 & 0 & 0 \\
0 & 0 & \frac{\mathrm{d} \tau(t)}{\mathrm{d} t}-1 & 0 \\
0 & 0 & 0 & \frac{\mathrm{d} \tau(t)}{\mathrm{d} t}-1
\end{array}\right]
$$

$Z_{32}$

$$
=\left[\begin{array}{llll}
p_{11}-p_{51} & p_{12}-p_{52} & p_{13}-p_{53} & p_{14}-p_{54} \\
p_{21}-p_{61} & p_{22}-p_{62} & p_{23}-p_{63} & p_{24}-p_{64} \\
p_{31}-p_{71} & p_{32}-p_{72} & p_{33}-p_{73} & p_{34}-p_{74} \\
p_{41}-p_{81} & p_{42}-p_{82} & p_{43}-p_{83} & p_{44}-p_{84}
\end{array}\right]
$$

$$
\begin{aligned}
& Z_{33} \\
& =\left[\begin{array}{cccc}
p_{15}-p_{55} & p_{16}-p_{56} & p_{17}-p_{57} & p_{18}-p_{58} \\
p_{25}-p_{65} & p_{26}-p_{66} & p_{27}-p_{67} & p_{28}-p_{68} \\
p_{35}-p_{75} & p_{36}-p_{76} & p_{37}-p_{77} & p_{38}-p_{78} \\
p_{45}-p_{85} & p_{46}-p_{86} & p_{47}-p_{87} & p_{48}-p_{88}
\end{array}\right]
\end{aligned}
$$

Now we obtain the matrix $\delta(\theta+\tau(t), \sigma+\tau(t))$,

$$
\begin{aligned}
& \delta_{11}(\theta+\tau(t), \sigma+\tau(t)) \\
& =\frac{b_{11}}{1-\frac{\mathrm{d} \tau(t)}{\mathrm{d} t}} \beta_{11}(\theta-\sigma)+\frac{b_{21}}{1-\frac{\mathrm{d} \tau(t)}{\mathrm{d} t}} \beta_{21}(\theta-\sigma) \\
& \delta_{12}(\theta+\tau(t), \sigma+\tau(t)) \\
& =\frac{b_{11}}{1-\frac{\mathrm{d} \tau(t)}{\mathrm{d} t}} \beta_{12}(\theta-\sigma)+\frac{b_{21}}{1-\frac{\mathrm{d} \tau(t)}{\mathrm{d} t}} \beta_{22}(\theta-\sigma) \\
& \delta_{21}(\theta+\tau(t), \sigma+\tau(t)) \\
& =\frac{b_{12}}{1-\frac{\mathrm{d} \tau(t)}{\mathrm{d} t}} \beta_{11}(\theta-\sigma)+\frac{b_{22}}{1-\frac{\mathrm{d} \tau(t)}{\mathrm{d} t}} \beta_{21}(\theta-\sigma) \\
& \delta_{22}(\theta+\tau(t), \sigma+\tau(t)) \\
& =\frac{b_{12}}{1-\frac{\mathrm{d} \tau(t)}{\mathrm{d} t}} \beta_{12}(\theta-\sigma)+\frac{b_{22}}{1-\frac{\mathrm{d} \tau(t)}{\mathrm{d} t}} \beta_{22}(\theta-\sigma)
\end{aligned}
$$

for $t \geq t_{0}, \theta \in[-\tau(t), 0], \sigma \in[\theta, 0]$, where $0 \leq \tau(t) \leq r$.

Figures 3-7 show graphs of the functions $\alpha(t), \beta(\xi)$, obtained with a Matlab code, for the following matrices 
$A, B, W$ of the system 52 :

$$
\begin{aligned}
A & =\left[\begin{array}{ll}
-1 & 0.3 \\
0.5 & -2
\end{array}\right], \quad B=\left[\begin{array}{cc}
-1 & 0.4 \\
0.1 & -1
\end{array}\right], \\
W & =\left[\begin{array}{ll}
1 & 1 \\
0 & 1
\end{array}\right]
\end{aligned}
$$

and a time delay given by the function

$$
\tau(t)=r\left(1-\exp \left(-\frac{t}{T}\right)\right),
$$

where $r=0.5, T=1$.

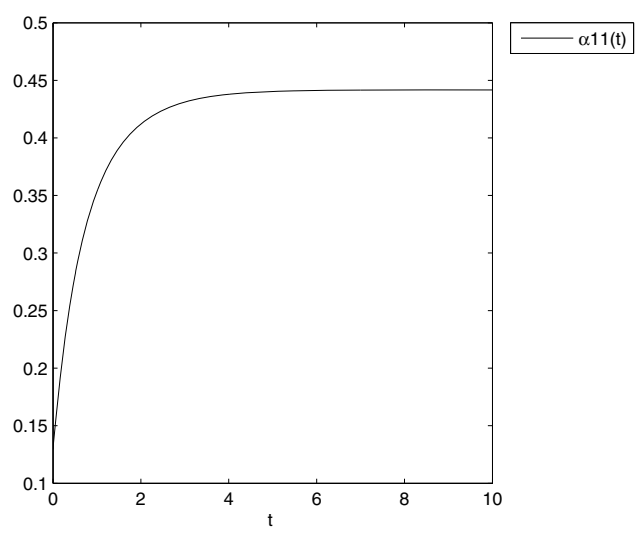

Fig. 3. Parameter $\alpha_{11}(t)$.

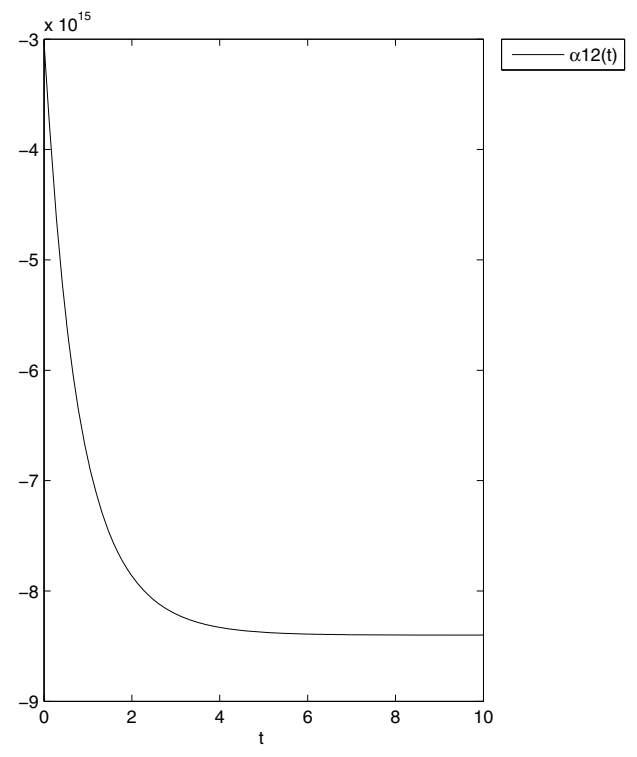

Fig. 4. Parameter $\alpha_{12}(t)$.

The matrix $\alpha(t)$ is positive definite for $r \leq 0.86$, so we have obtained a stability region for the system (52).

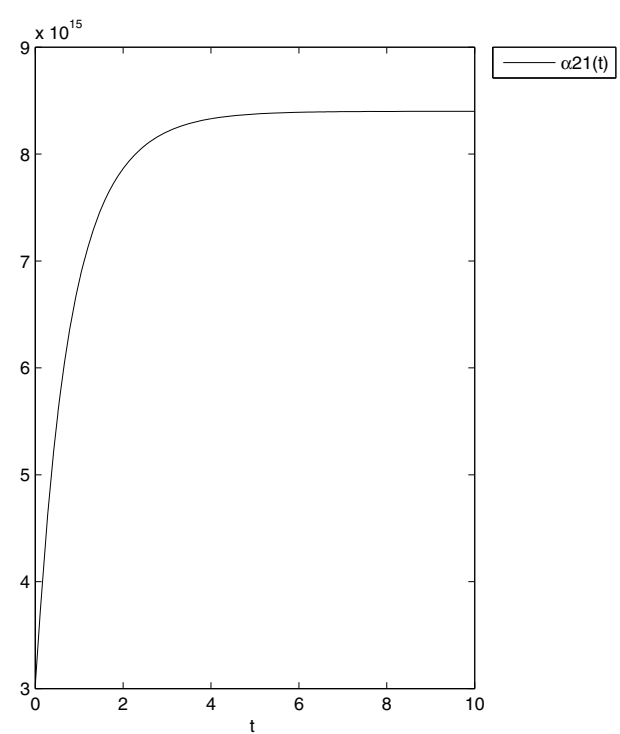

Fig. 5. Parameter $\alpha_{21}(t)$.

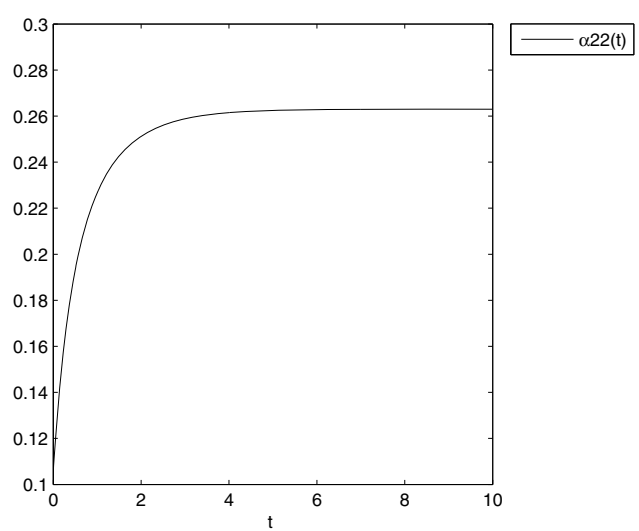

Fig. 6. Parameter $\alpha_{22}(t)$.

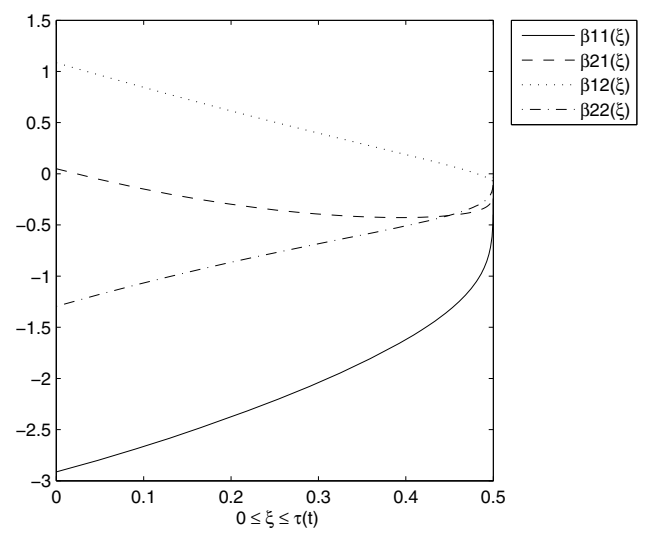

Fig. 7. Elements of the matrix $\beta(\xi)$. 


\section{Conclusions}

The paper presented the procedure of determining the coefficients of the Lyapunov functional given by the formula (4) for a linear system with an interval time-varying delay, described by Eqn. (1). This article extends the method presented by Repin to the system with an interval timevarying delay. The presented method allows achieving analytical formulas on the coefficients of the Lyapunov functional which can be used to examine the stability of time delay systems with an interval time-varying delay and in the process of parametric optimization for calculation of the square performance index given by the formula (7).

\section{Acknowledgment}

The author wishes to thank the editors and the reviewers for their suggestions, which have improved the quality of the paper.

\section{References}

Duda, J. (1986). Parametric Optimization Problem for Systems with Time Delay, Ph.D. thesis, AGH University of Science and Technology, Cracow.

Duda, J. (1988). Parametric optimization of neutral linear system with respect to the general quadratic performance index, Archiwum Automatyki i Telemechaniki 33(3): 448-456.

Duda, J. (2010a). Lyapunov functional for a linear system with two delays, Control \& Cybernetics 39(3): 797-809.

Duda, J. (2010b). Lyapunov functional for a linear system with two delays both retarded and neutral type, Archives of Control Sciences 20(LVI): 89-98.

Fridman, E. (2001). New Lyapunov-Krasovskii functionals for stability of linear retarded and neutral type systems, Systems \& Control Letters 43(4): 309-319.

Górecki, H., Fuksa, S., Grabowski, P., Korytowski, A. (1989). Analysis and Synthesis of Time Delay Systems, John Wiley \& Sons, Chichester/New York, NY/Brisbane/Toronto/Singapore.

Gu, K. (1997). Discretized LMI set in the stability problem of linear time delay systems, International Journal of Control 68(4): 923-934.

Gu, K. and Liu, Y. (2009). Lyapunov-Krasovskii functional for uniform stability of coupled differential-functional equations, Automatica 45(3): 798-804.

Han, Q.L. (2004). On robust stability of neutral systems with time-varying discrete delay and norm-bounded uncertainty, Automatica 40(6): 1087-1092.

Han, Q.L. (2004). A descriptor system approach to robust stability of uncertain neutral systems with discrete and distributed delays, Automatica 40(10): 1791-1796.

Han, Q.L. (2005). On stability of linear neutral systems with mixed time delays: A discretised Lyapunov functional approach, Automatica 41(7): 1209-1218.
Han, Q.L. (2009). A discrete delay decomposition approach to stability of linear retarded and neutral systems, Automatica 45(2): 517-524.

Infante, E.F. and Castelan, W.B. (1978). A Lyapunov functional for a matrix difference-differential equation, Journal of Differential Equations 29: 439-451.

Ivanescu, D., Niculescu, S.I., Dugard, L., Dion, J.M. and Verriest, E.I. (2003). On delay-dependent stability for linear neutral systems, Automatica 39(2): 255-261.

Kharitonov, V.L. (2005). Lyapunov functionals and Lyapunov matrices for neutral type time delay systems: A single delay case, International Journal of Control 78(11): 783800.

Kharitonov, V.L. (2008). Lyapunov matrices for a class of neutral type time delay systems, International Journal of Control 81(6): 883-893.

Kharitonov, V.L. and Hinrichsen, D. (2004). Exponential estimates for time delay systems, Systems \& Control Letters 53(5): 395-405.

Kharitonov, V.L. and Plischke, E. (2006). Lyapunov matrices for time-delay systems, Systems \& Control Letters 55(9): 697 706.

Kharitonov, V.L., Zhabko, A.P. (2003). Lyapunov-Krasovskii approach to the robust stability analysis of time-delay systems, Automatica 39(1): 15-20.

Klamka, J. (1991). Controllability of Dynamical Systems, Kluwer Academic Publishers, Dordrecht.

Repin, Yu. M. (1965). Quadratic Lyapunov functionals for systems with delay, Prikladnaja Matiematika i Miechanika 29: $564-566$.

Respondek, J.S. (2008). Approximate controllability of the $n$-th order infinite dimensional systems with controls delayed by the control devices, International Journal of Systems Science 39(8): 765-782.

Richard, J.P. (2003). Time-delay systems: An overview of some recent advances and open problems, Automatica 39(10): $1667-1694$.

Wang, D., Wang, W. and Shi, P. (2009). Exponential H-infinity filtering for switched linear systems with interval timevarying delay, International Journal of Robust and Nonlinear Control 19(5): 532-551.

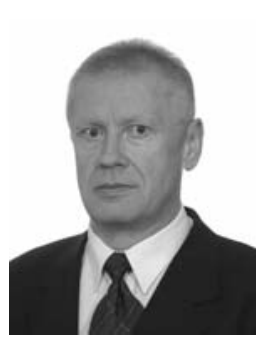

Józef Duda graduated from the Faculty of Electrical Engineering, Automatics and Electronics, AGH University of Science and Technology in Cracow, Poland. He received an M.Sc. in electronics/automatic control in 1981. After completing doctoral studies, he received his Ph.D. degree in automatic control from the AGH University in 1986. His main research concerns time delay systems and application of Lyapunov functionals to stability analysis of time delay systems and in the process of parametric optimization.

Received: 27 January 2011 Revised: 26 May 2011

Re-revised: 1 August 2011 